\title{
OVERALL SHAPE OPTIMIZATION OF OFFSHORE OIL REMOVAL TANKER BASED ON FLUENT
}

\author{
Ying Lin ${ }^{1}$, Shan Guo ${ }^{1}$, Peng Xu ${ }^{2}$, Hong Lin ${ }^{1}$, Chen Wang ${ }^{1}$, Mengqiao Luo ${ }^{1}$, Maohua Xiao ${ }^{1 a^{*}}$ \\ ${ }^{1}$ College of Engineering, Nanjing Agricultural University, Nanjing, 210031, China \\ 2 Dongtai Jinjin Fruit Industry Co., Ltd., Yan Cheng, 224200, China \\ Email: xiaomaohua@njau.edu.cn
}

\begin{abstract}
In recent years, marine oil spill accidents have occurred frequently. A large number of scholars at home and abroad have conducted in-depth theoretical and experimental research on offshore oil removal tankers. However, the research on its full-scale driving stability and drag reduction performance was still insufficient. The overall efficiency of the oil removal tanker was low, and its resource utilization rate was not high. In this paper, an optimization method based on the whole modelling of the oil removal tanker was proposed. The CFD computational fluid dynamics method was adopted, which has been verified by validity. By studying the optimization plan of the overall shape, the overall work efficiency and the drag reduction performance of the oil removal tanker could be improved, and the energy could be saved. The final drag reduction was reached up to $515.86 \mathrm{kN}$, a decrease of about $21.76 \%$, and the drag coefficient was reduced by 0.479. This paper provided an important reference for the research and development of the offshore oil removal tanker by the optimization method of the oil removal tanker and the overall shape optimization scheme of the oil removal tanker on the sea.
\end{abstract}

Keywords: Drag reduction performance; FLUENT; Free surface; Oil removal tanker; Overall shape.

\section{Introduction}

CFD technology can play an important role in the research of ship resistance performance, ship-shape optimization, and even a wider range of ship hydrodynamic performance. It can achieve more goals that can only be achieved by experiments at present [1]. In recent years, many experiments have proved the reliability and accuracy of CFD, and some achievements have been made in the study of ship resistance performance by using computational fluid dynamics, which is more and more favoured by researchers[2].

Oil leakage into the sea will not only waste scarce oil resources and cause huge economic losses, but also cause serious damage to the marine ecological environment and seriously affect the production and life of coastal people [3]. In recent years, a large number of scholars at home and abroad have carried out in-depth research on the theory and experiment of offshore oil removal tankers[4]. Due to functional reasons, oil removal tankers are different from ordinary ships in design [5]. Complicated geometric shape, large changes in surface curvature, and mutual interference between different components of oil removing tankers resulted in more and more prominent problems such as the low overall efficiency of the oil removal tankers and the low utilization of resources [6].
Liu from Shenyang University of Technology designed a kind of oil removing ship, using remotecontrol technology to optimize the oil removing ship, which reduced labour costs [7]. But this optimization method made the oil removal tanker could only be carried out in a narrow space oil removing operation. Qin from Chongqing Jiaotong University put forward a trident suction tanker optimization method to improve the economy and reliability of the tanker [8]. But this was only a preliminary study, which was still one kind of immature technology. Cheng et al. from Shandong Jiaotong University developed a dynamic cyclone for oil-containing sewage, which improved the efficiency of the tanker. But it was only a local design of the tanker, not based on the whole[9][10].

Most of the above research is to improve the hull structure of the oil removal tanker and improve its local working performance through experimental verification, which has certain limitations [11]. Due to the complexity of hull processing technology, there is little research on the overall modelling and drag reduction performance of oil removal tankers[12]. At the same time, under the background of the rapid development of CFD technology, aiming at the deficiency of existing research, this paper puts forward a systematic and general optimization method based on the whole modelling of oil remover. 
By using the software of FLUENT, we analyze the surface pressure nephogram of a newly invented oil remover and the numerical simulation results of the newly invented oil remover in full scale combined with wave resistance and free surface wave conditions, so as to raise the overall working efficiency of oil remover, improve the drag reduction performance of oil remover and increase the utilization rate of resources at the same time. It provides an important reference for the research and development of offshore oil removal tankers.

\section{Geometric Model of Offshore Oil Removal Tanker}

The main parameters should be determined first when studying the flow field distribution of the offshore oil removing tanker. The oil-removing tanker adopted in this paper is a new type of oilremoving tanker. Its shape is quite different from the generalship structure due to the specially designed oil-absorbing and storage structure. At the beginning of the design, there are two design schemes A and B for the offshore oil removing tanker, and the main parameters and calculation conditions are as follows:
Table 1. Main parameters of the oil removal tanker

\begin{tabular}{|l|l|r|r|r|}
\hline $\begin{array}{l}\text { Ship } \\
\text { type }\end{array}$ & $\begin{array}{l}\text { General } \\
/ \mathrm{m}\end{array}$ & $\begin{array}{l}\text { Molded } \\
\text { breadth } \\
\text { /m }\end{array}$ & $\begin{array}{l}\text { Molded } \\
\text { depth/m }\end{array}$ & $\begin{array}{r}\text { Design } \\
\text { draft/m }\end{array}$ \\
\hline $\begin{array}{l}\text { Type } \\
\text { A }\end{array}$ & 45.649 & 40.127 & 9.216 & 4 \\
\hline $\begin{array}{l}\text { Type } \\
\text { B }\end{array}$ & 54.156 & 52.148 & 9.519 & 4 \\
\hline
\end{tabular}

The above two designs $\mathrm{A}$ and $\mathrm{B}$ of the new degreaser are shown in Figure 1:

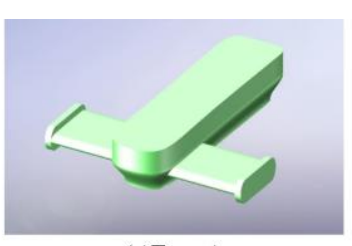

(a)Type $\mathrm{A}$

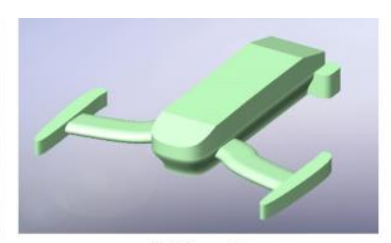

(b)Type B
Figure 1: Type $A$ and $B$ oil removing tankers

\section{Basic Theory of Numerical Simulation 3.1 Governing Equation}

The governing equations for the calculation of this new tanker's whole flow field include the incompressible fluid continuity equation and the RANS equation. The continuity equation and the RANS equation are shown in Eq. (1) and Eq. (2):

$$
\begin{gathered}
\frac{\partial \bar{u}_{i}}{\partial x_{i}}=0, \\
\rho \frac{\partial \bar{u}_{i}}{\partial t}+\rho \bar{u}_{j} \frac{\partial \bar{u}_{i}}{\partial x_{j}}=-\frac{\partial \bar{p}}{\partial x_{i}}+\mu \frac{\partial^{2} \bar{u}_{i}}{\partial x_{j} \partial x_{j}}-\rho \frac{\partial \bar{u}_{i}^{\prime} \partial \bar{u}_{j}^{\prime}}{\partial x_{j}}+\rho \overline{f i},
\end{gathered}
$$

where $\bar{u}_{i}$ is the average Reynolds velocity, $\bar{u}_{j}$ is the fluctuating velocity, and $\rho \overline{u_{u}^{t} u_{j}^{t}}$ is the Reynolds stress [13].

\subsection{Turbulence Model}

The RNG k- $\varepsilon$ model shows the effects of small-scale motions through the large-scale motions and the modified viscosity terms so that these small-scale

motions are systematically removed from the governing equations. The transport equations corresponding to $\mathrm{k}$ and $\varepsilon$ are shown in eq. (3) and eq. (4):

$$
\begin{gathered}
\frac{\partial(\rho k)}{\partial t}+\frac{\partial\left(\rho k u_{i}\right)}{\partial x_{i}}=\frac{\partial}{\partial x_{j}}\left[\alpha_{k} \mu_{e f f} \frac{\partial k}{\partial x_{j}}\right]+G_{k}+\rho \varepsilon \\
\frac{\partial(\rho \varepsilon)}{\partial t}+\frac{\partial\left(\rho \varepsilon u_{i}\right)}{\partial x_{i}}=\frac{\partial}{\partial x_{j}}\left[\alpha_{\varepsilon} \mu_{e f f} \frac{\partial \varepsilon}{\partial x_{j}}\right]+C_{1 s}^{*} \frac{\varepsilon}{k} G_{k}-C_{2 \varepsilon} \rho \frac{\varepsilon^{2}}{k}
\end{gathered}
$$

The detailed process and parameters can be seen in reference [14]. Compared with the standard $\mathrm{k}-\varepsilon$ model, the RNG $\mathrm{k}-\varepsilon$ model adds a condition to the $\varepsilon$ equation, which effectively improves the accuracy[15]. The RNG k- $\varepsilon$ turbulence model is used to simulate the wave-making resistance of the free liquid surface for the tanker described in this paper, which has higher reliability and accuracy.

\subsection{Volume of Fluid Function Method (VOF)}

The basic principle of the VOF method is to determine the free surface by studying the fluid and the mesh volume ratio function $F$ in the grid element, and to track the change of the fluid, rather than track the motion of particles on the free liquid surface. 
The VOF method can deal with strong nonlinear phenomena such as free surface reentry, which requires short calculation time and less storage. The VOF method constructs and tracks free surfaces according to the volume ratio function $F$. The detailed methods and principles can be seen in the literature [9]. The tanker in this paper belongs to the interface simulation between two incompatible liquids, so the VOF model is used for the simulation.

The VOF model is used to describe the changing process of the free liquid level and to simulate two or more unmixable fluids.

In each control volume, the sum of all phase volume fractions is 1 , i.e. $\sum \alpha_{q}=1$. By solving the continuous equation of single-phase or multiphase volume ratio, the interface between phases can be tracked. For phase $\mathrm{q}$, the transport equation is shown in Eq. (5) :

$$
\frac{\partial \alpha_{q}}{\partial t}+\vec{v} \cdot \nabla \alpha_{q}=0
$$

The properties that appear in the transport equation are determined by the phases present in each control volume. For example, for a two-phase flow, if the volume ratio of the second phase is tracked, then the density in each cell: $\rho=\alpha_{2} \rho_{2}+\left(1-\alpha_{1}\right) \rho_{1}$. For an N-phase system, the volume ratio averages density: $\rho=\sum \alpha_{q} \rho_{q}$. By solving a single momentum equation in the entire region, the resulting velocity vector field is shared by all phases, and the momentum equation depends on the volume ratio of all phases through the attribute sum, as shown in Eq. (6):

$$
\frac{\partial}{\partial t}(\rho \vec{v})+\nabla \cdot\left(\rho \vec{v}^{2}\right)=-\nabla P \cdot\left(\mu\left(\nabla \vec{v}+\nabla v^{T}\right)\right)+\rho \vec{g}+\vec{F}
$$

where $\vec{v}$ is the velocity field of the fluid, $\vec{g}$ is the acceleration of gravity, $\vec{F}$ is the volume force exerted by the microelement and $\vec{v}^{T}$ is the transpose matrix of the velocity field. The letters with arrows in the equation represent the vector expression of the physical quantity in the equation[16][18].

\section{Analysis of External Flow Field and Overall Optimization of Shipshape 4.1 Pretreatment of Flow Field Model}

After determining the flow field of the two types of oil removing tankers, ANSYS ICEM-CFD was used for meshing, and the mesh was encrypted around the hull and on the free liquid surface to obtain a more accurate calculation result. The numerical simulation of the oil removal tanker uses a threedimensional, unsteady model, the VOF two-phase flow calculation model, and the RNG k- $\varepsilon$ turbulence model. The calculation method uses the PISO algorithm, and the calculation accuracy is secondorder.

\subsection{Analysis of Calculation Results 4.2.1 Numerical simulation analysis of tanker hull}

The analysis results of FLUENT were checked in the CFD-post processing. This paper focuses on comparing the flow field characteristics and drag reduction performance of oil removal tankers with different shapes in the same design, so only the simulation results under $4,6,8,10 \mathrm{~m} / \mathrm{s}$ are simulated to reduce the calculation time.

The numerical simulation of the oil removal tanker uses a three-dimensional, unsteady model, the VOF two-phase flow calculation model, and the RNG k- $\varepsilon$ turbulence model. The calculation method uses the PISO algorithm, and the calculation accuracy is second-order. The boundary conditions are set as follows: the velocity inlet is adopted, the pressure outlet is adopted for the exit boundary, and the time discretization is $0.005 \mathrm{~s}$. The calculated results are saved every 0.1 seconds, and the calculated results of resistance and resistance coefficient values are averaged between 400 and 500 steps. Considering the existence of error, the average value between 200 and 400 steps is selected if the individual data is not ideal.

Table 2 shows the calculation results of resistance and resistance coefficient, and Figure 2 shows the comparison diagram of resistance curves of two different shapes of the same oil removal tanker. It can be seen that with the increase of speed, the resistance of the tanker shows an upward trend, and the shape of resistance curves of two shapes of the same tanker is close. The drag of the type A tanker is larger than that of type B, whose drag coefficient is also larger. Based on the analysis data of the two types of the tanker and the analysis of the relevant knowledge of ship mechanics, the overall shape designs of these two types are not reasonable, and the drag reduction effect is not obvious: 
Table 2. Calculation results of resistance and resistance coefficient

\begin{tabular}{|c|c|c|c|}
\hline Speed $/(\mathrm{m} / \mathrm{s})$ & Type of tanker & The drag coefficient & Resistance $/ \mathrm{kN}$ \\
\hline \multirow{2}{*}{4} & A & 0.307 & 192.53 \\
\cline { 2 - 4 } & B & 0.100 & 84.39 \\
\hline \multirow{2}{*}{6} & A & 0.507 & 711.98 \\
\cline { 2 - 4 } & B & 0.258 & 488.58 \\
\hline \multirow{2}{*}{8} & A & 0.597 & 1492.93 \\
\cline { 2 - 4 } & B & 0.378 & 1272.45 \\
\hline \multirow{2}{*}{10} & A & 0.606 & 2370.25 \\
\cline { 2 - 4 } & B & 0.396 & 2087.44 \\
\hline
\end{tabular}

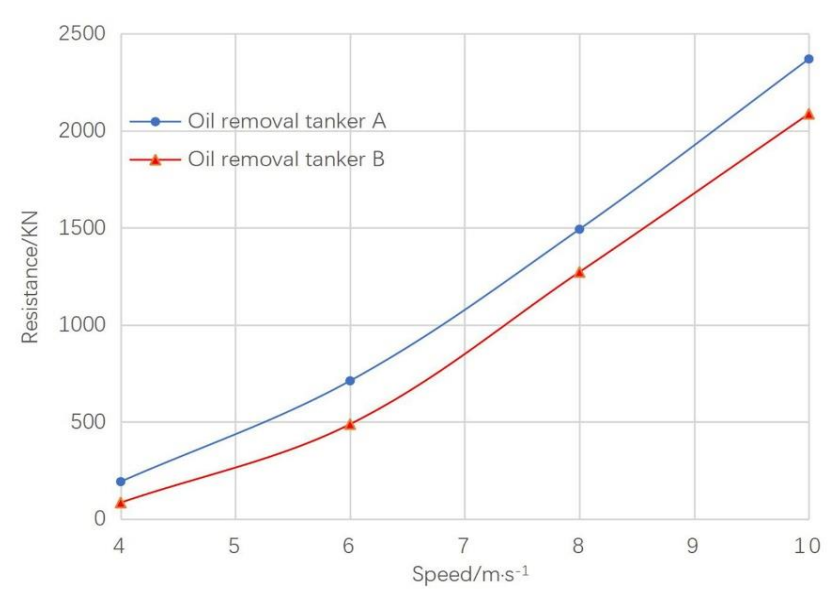

Figure 2: Comparison of resistance curves of different shapes

The following is a macro analysis under a certain working condition at a certain moment. Figure 3 shows the hull pressure cloud diagram of the two configurations of the oil removal tanker with a speed of $6 \mathrm{~m} / \mathrm{s}$. Figure 4 shows the velocity vector diagram of the symmetry plane of $10 \mathrm{~m} / \mathrm{s}$ and the locally enlarged diagram of the bow:
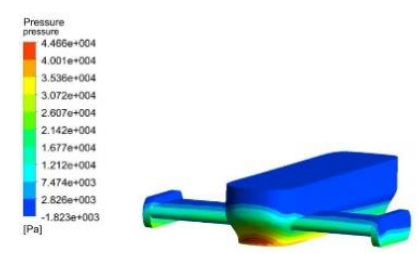

(a)Type A

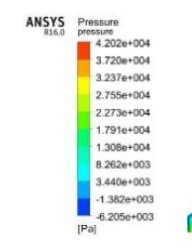

$\perp$

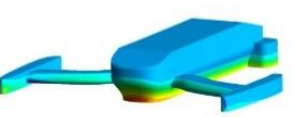

$$
\text { (b) Type B }
$$

ANSYS

$\stackrel{1 .}{.}$

Figure 3: Pressure cloud diagram of type A and type B tankers

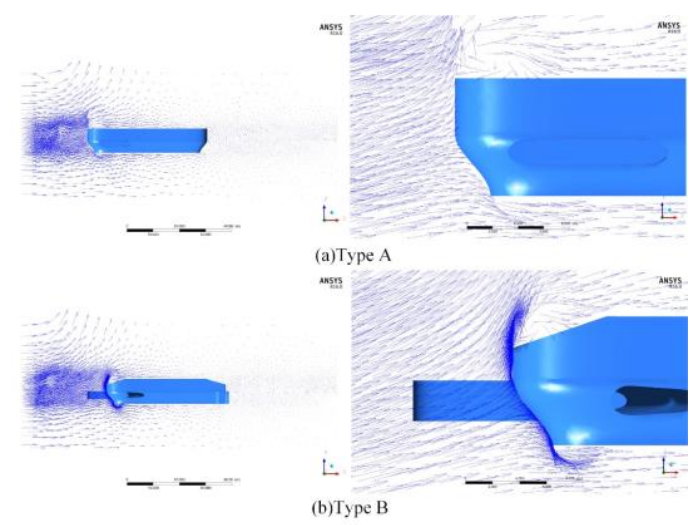

Figure 4: Speed vector diagram of symmetry plane of type A and type B tanker and partial enlargement of bow 
According to the pressure cloud diagram, the hydrostatic pressure distribution from the bow to the stern of the two types of tankers is basically the same, and it can be observed that the surface pressure located below the bow is concentrated, which is respectively $44660 \mathrm{~Pa}$ and $42020 \mathrm{~Pa}$. This position is the largest part of the surface pressure of the whole hull structure, which requires a high strength for the bow. As the incoming liquid meets the front end of the tanker, the incoming flow is blocked and its speed is reduced, with a vortex formed. The flow is too late to turn and partially separates. From the velocity vector diagram in Figure 4, it is observed that the fluid separation point of $\mathrm{A}$ is located at the upper edge of the bow, and the air separation point of $B$ is located at the upper and lower edges of the bow. Due to the improper geometric design of the overall shape of the oil removing tanker, the fluid cannot continue to flow smoothly. It can be found from the streamline distribution that the internal vortex system at the fluid separation site is complex, forming a turbulent flow zone.

Combined with the calculation results of drag and drag coefficient in Figure 2, it can be concluded that the two types of tankers at different speeds have large hull drag and drag coefficients, poor drag reduction performance, and unreasonable overall shape design. This paper makes a comprehensive comparison between the two types of tankers and improvement.

\subsubsection{Overall Optimization and Analysis of Ship Type}

From the above data analysis, it can be seen that the resistance, resistance coefficient, pressure, etc. of the two models of oil removal tankers are relatively large. The overall shape design is not scientific enough, and there is a waste of energy and low oil removal efficiency. In this paper, from the aspect of improving drag reduction performance, considering the influence of man-machine engineering and material curvature on strength, as well as the influence of manoeuvring, waveform, general layout, the overall type of tanker is analyzed and improved, mainly including: streamline structure design of the oil removal tanker, optimization of the windward structure of the bow of the oil removal tanker, surface design[19][20].

According to the analysis of numerical simulation results of tanker A and tanker B, the large frontal windward area of the bow leads to high pressure, which is easy to form vortices. Due to improper geometric structure design, the airflow and fluid separation points of tanker A and tanker B appear prematurely, forming a turbulent region. Therefore, the frontal windward area of the bow is reduced, and the curved surface of the bow is smoothed to make the contour line design of the tanker more rounded. At the same time, we improve the ship's surface, so that the transition is smooth. Remove the abrupt and depressing parts of the oil removal tanker surface to avoid the large abrupt change in the direction of each turning surface of the ship. Improve the structure of the left and right flanks of the tanker to delay the fluid separation point[21][23].

Considering the requirement of the function of the oil removing tanker, the length $\mathrm{L}$ is set to $\mathbf{5 4 . 3 2 9}$ $\mathrm{m}$. The depth D should not be too small and must meet the minimum freeboard requirements. If the depth $\mathrm{D}$ is too large, the centre of gravity will increase, which will affect the slewing performance during high-speed navigation and is unfavourable for fast navigation. Under comprehensive consideration, the depth $\mathrm{D}$ should be set at $10.434 \mathrm{~m}$. The width of the ship mainly affects the position of the metacentric of the ship, the buoyancy of the ship, and the size of the tank capacity of the ship. From the perspective of the speed, keeping other ship type parameters unchanged, reducing the width of the small ship can increase the speed. The maximum width B selected here is $40.201 \mathrm{~m}$.

At the same time, it is not difficult to see that the overall shape design uses the advantages of the sharks. Sharks have a very streamlined shape, causing only small fluid fluctuations, reducing the generation of eddy currents. Based on this configuration, the overall shape of the ship is optimized.

The optimized shape structure of the tanker is shown in Figure 5, and the detailed design is shown in the references and patents [23].
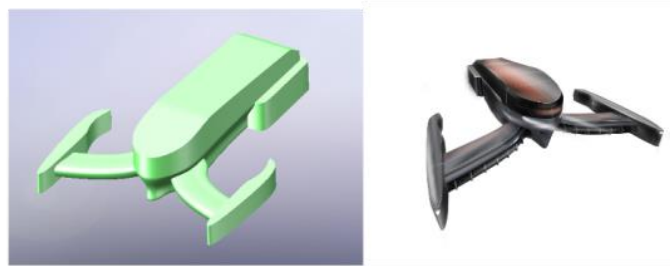

Figure 5: Oil removal tanker after optimization

\section{Analysis and Comparison of the Results After Optimization of the Overall Modeling \\ 5.1 Pretreatment for Numerical Simulation of oil Removal Tanker}

\subsubsection{Determination of flow field calculation domain}

Use the ANSYS designmodeler module to import the model and determine the fluid control domain (Figure 6). If the domain is too large, the number of computing grids is huge, which increases unnecessary computation. If the area is too small, the calculation accuracy cannot be satisfied [24]. 
Based on the above considerations, the calculation domain is: the longitudinal direction is approximately 0.5 times the length of the tanker extending from the bow to the front, and the length of the basin extending from the bow to the back is 2 times the length of the tanker.
The lateral width is 2.5 times the width of the tanker. Vertically extending from the free liquid surface up to 5 times the draft as an air layer, the free liquid surface extends down 6 times the draft height. Since the tanker is symmetric, take one half of this domain for calculation [25].

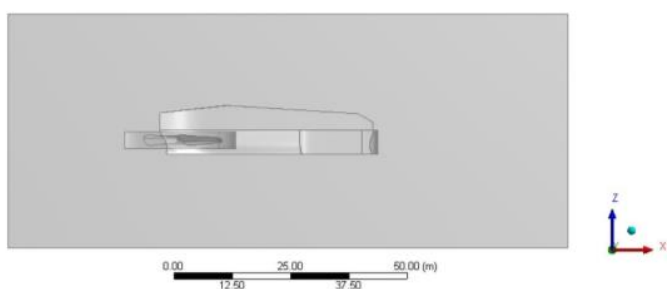

Figure 6: Schematic diagram of fluid control domain (take 1/2)

\subsubsection{Grid generation}

After importing the 3D calculation model generated by PTC CREO through the conversion file format, it is encrypted based on curvature and approximation. In order to analyze the wavemaking situation of the free liquid surface and the pressure distribution of the hull surface, mesh encryption is carried out around the hull and the free liquid surface to obtain more accurate calculation results.

After optimizing, the number of grids is about $1.8 \times 10^{6}$, and the maximum skewness value of the grid is about $0.87<0.95$, which is an acceptable quality level. Figure 7 shows the grid division of the optimized tanker and its computing domain:

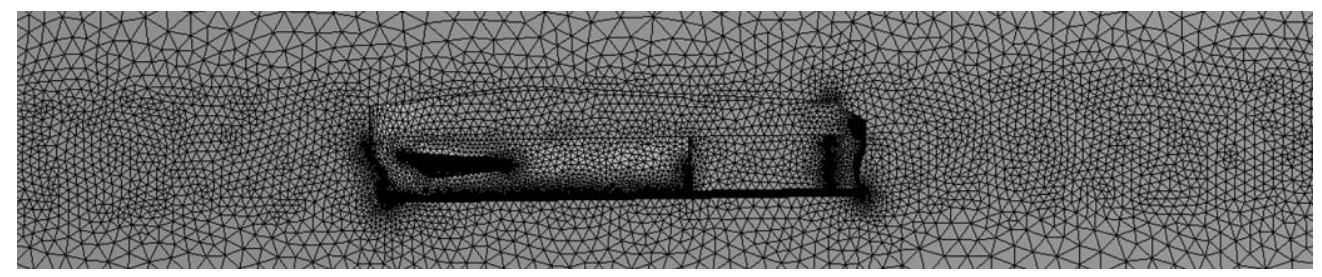

Figure 7: Local diagram of optimized oil removal tanker grid

\subsubsection{Solver parameters such as boundary conditions and turbulence modes}

The numerical simulation of the oil removal tanker uses a three-dimensional, unsteady model, the VOF two-phase flow calculation model, and the RNG k- $\varepsilon$ turbulence model. The calculation method uses the PISO algorithm, and the calculation accuracy is second-order.

The boundary conditions are set as follows: the velocity inlet is adopted, the pressure outlet is adopted for the exit boundary, and the time discretization is $0.005 \mathrm{~s}$. The calculated results are saved every 20 steps, and the calculated results of resistance and resistance coefficient are averaged between 400 and 500 steps. Considering the existence of error, the average value between 200 and 400 steps is selected if the individual data is not ideal.

\subsection{Comparison and Analysis of Numerical Simulation Results of oil Removing Tankers 5.2.1 Numerical simulation results of the hull of the tanker}

The post-processing software CFD-POST was used to view the analysis results of the optimized oil removal tanker, and the pressure cloud map was obtained, as shown in Figure 8. The maximum pressure of the optimized ship type is $41840 \mathrm{~Pa}$, which is less than the maximum pressure of $44660 \mathrm{~Pa}$ of oil removing tanker before optimization. At the same time, it can be seen that the surface pressure of the optimized bow is no longer concentrated in the bow, but dispersed in the part of the hull that is in contact with water at the same time, which indicates that it has a good overall optimization effect on the tanker.

Figure 9 is the vector diagram of the speed of the fluid domain's symmetry plane before and after optimization. The fluid separation point of $A$ is located at the upper edge of the bow, and the air separation point of $\mathrm{B}$ is located at the upper and lower edges of the bow. Due to improper geometric structure design, the fluid cannot continue to flow smoothly. From the streamline distribution, it can be found that the internal vortex system at the fluid separation site is complex, forming a turbulent zone. And the smooth and continuous shape of the optimized tanker can guide the fluid well and make the fluid flow smoothly close to the surface of the hull. Compared with the tanker before optimization, the curvature of the front end of the optimized 
tanker is greatly reduced, and the frontal windward area is also greatly reduced, making the streamlines around the hull more compact and the degree of fluid separation greatly reduced.
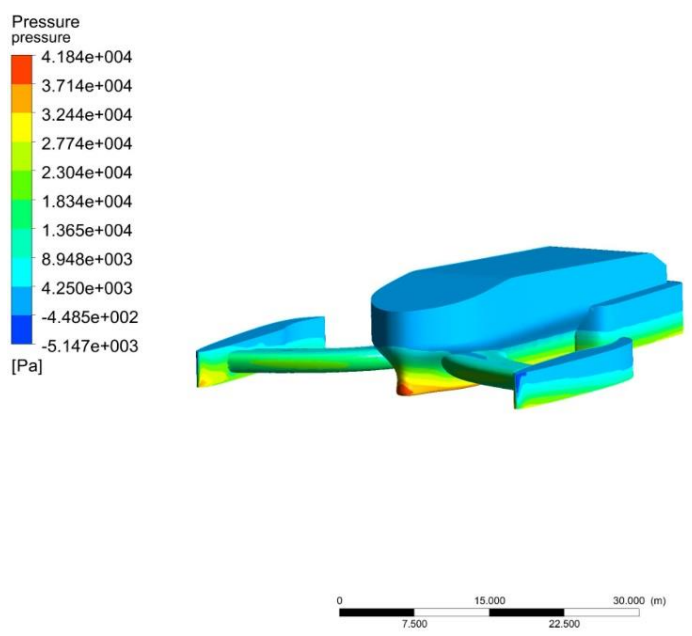

Optimized oil removal tanker
The drag reduction effect of the tanker is very significant, and the work efficiency is greatly improved.
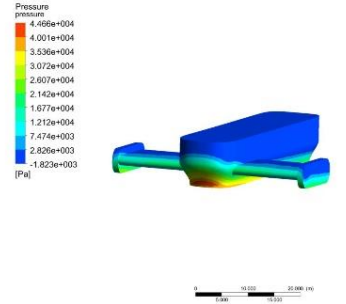

$\perp$

Oil removal tanker A

$\underset{\substack{\text { ANSYS } \\ \text { nla }}}{\text { and }}$
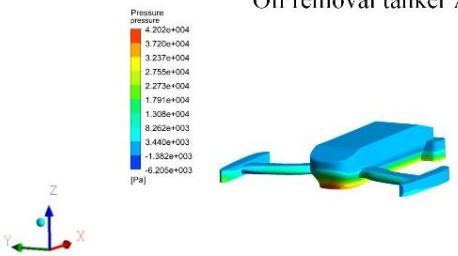

-

Oil removal tanker $\mathrm{B}$

Figure 8: Pressure cloud map of the optimized tanker

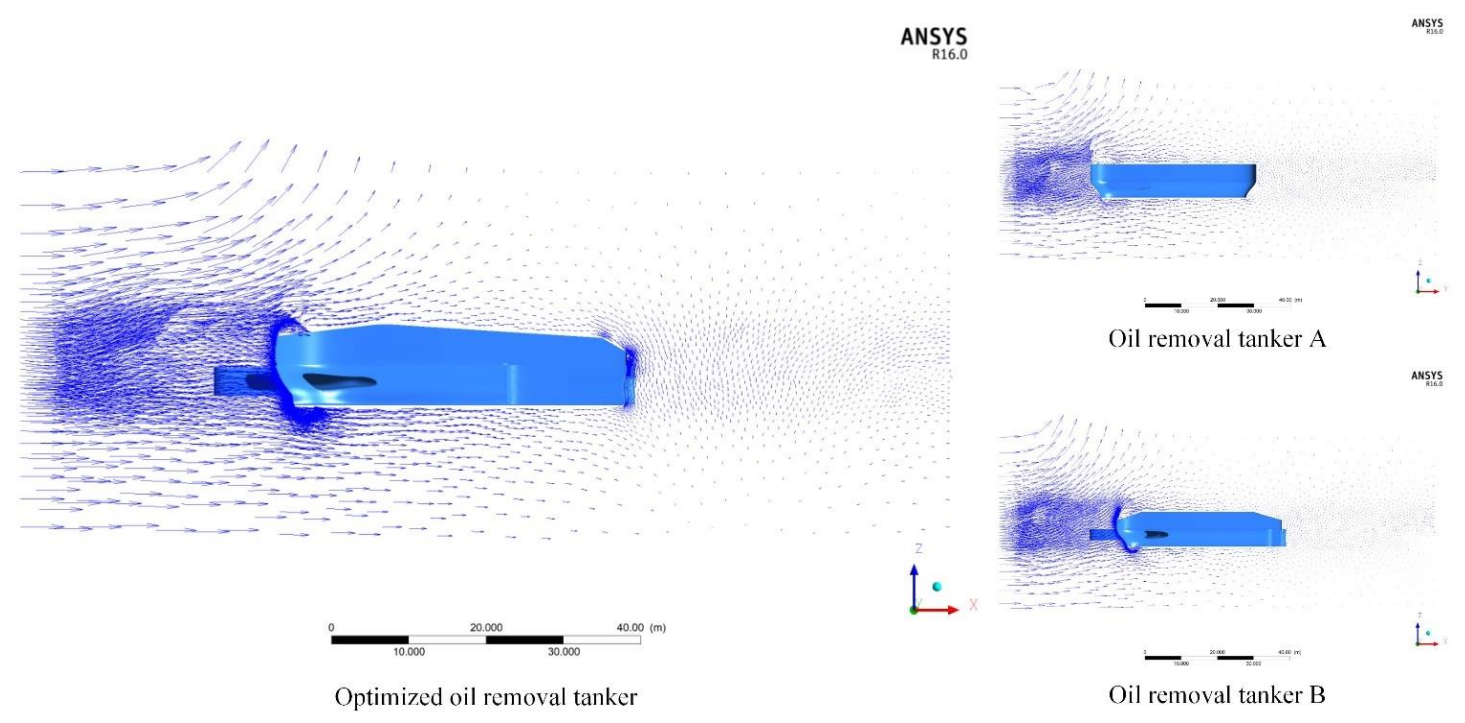

Figure 9: Comparison of velocity vectors of symmetry plane before and after tanker type optimization

Figure 10-(a) shows the comparison of the calculated results of $\mathrm{A}, \mathrm{B}$, and the optimized resistance of the tanker. As can be seen from the figure, the resistance of the tanker increases with the increase of working speed, which basically reflects the resistance characteristics of the calculated tankers. There is little difference between the resistance of $\mathrm{A}$ and $\mathrm{B}$ before the optimization, and the optimized ship can significantly improve the work efficiency, reduce the resistance and improve the drag reduction performance. After optimization, the resistance of the ship type can be greatly reduced: compared with the drag of the Type A tanker before optimization, the drag can be reduced by $515.86 \mathrm{kN}$, about 21.76\%; Compared with the Type B tanker before optimization, the drag can be reduced by $233.05 \mathrm{kN}$, about $11.16 \%$.

Figure 11-(b) shows the comparison of the calculated drag coefficient of type A, type B, and the optimized tanker, as shown in the figure: $C_{d}$ is the drag coefficient, $F_{r}$ is the Froude number, $F_{r}=\frac{V}{\sqrt{g L}}$. The figure shows that the optimized form drag coefficient was significantly less than A and B tanker before optimization, and the optimized at the same speed drag coefficient reduces the biggest 0.479 . Namely, the optimized overall modelling can reduce the total resistance hull and improve work efficiency, which further verified the effectiveness of the optimization method based on the overall shape of the tanker in this paper. 


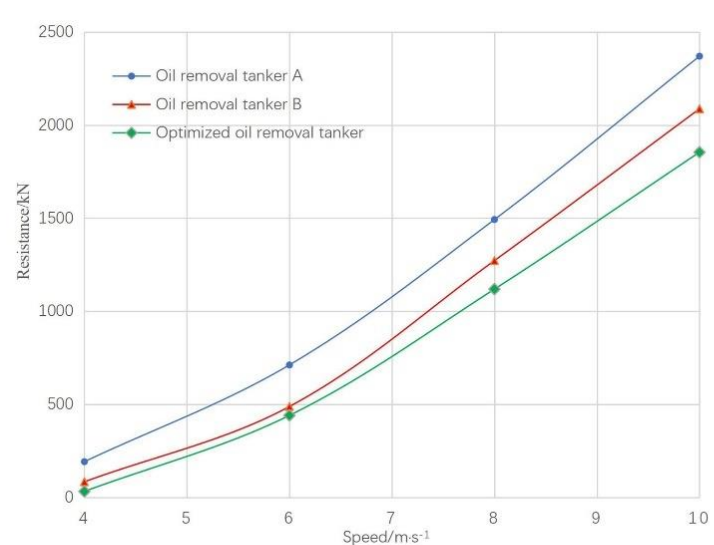

(a) Resistance-speed

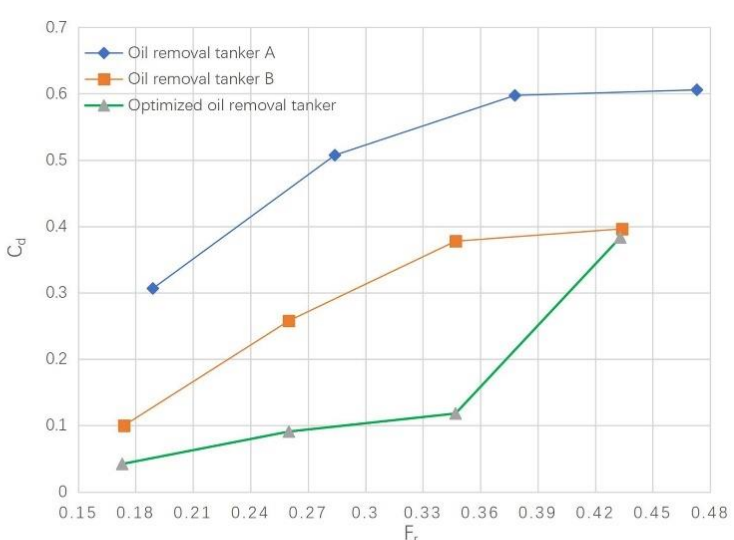

(b) Drag coefficient-Froude number

Figure 10: Comparison before and after optimization

\subsubsection{Numerical simulation results of free surface wave-making of oil-removing tanker}

The wave-making of the free liquid surface is an important standard to measure the overall shape of the tanker. The wave-making of the free liquid surface is simulated by using the VOF model, and the influence of the same velocity and different configurations on the wave-making resistance of the ship is analyzed.

After the calculation, the wave-making resistance value was read from ANSYS Fluent. Figure 11 shows the comparison of calculation results of Type $\mathrm{A}$ and $\mathrm{B}$ tanker and wave-making resistance obtained after optimization. It can be seen that the velocity is small when $F_{r}$ and the wave-making resistance is small. With the increase of the speed, the wave-making resistance increases, which is consistent with the following conclusions: when the ship is at low speed and low to medium speed, the proportion of wave-making resistance is not high compared with high speed; And as the speed increases to medium-to-high speed or high speed, the proportion of wave resistance increases rapidly, which leads to the increase of the influence of wave resistance on ship resistance[26]-[28]. It can be seen from Figure 11 that the wave-making resistance of the optimized tanker is lower than that of the optimized tanker type A and B, and the drag reduction performance of the optimized tanker is significantly improved: at medium and high speed, compared with the tanker type A, the wave-making resistance can be reduced by about $518.128 \mathrm{~N}$, about 21.95\%; Compared with the tanker B, the wave resistance can be reduced by about $234.374 \mathrm{~N}$, which is about $11.29 \%$.

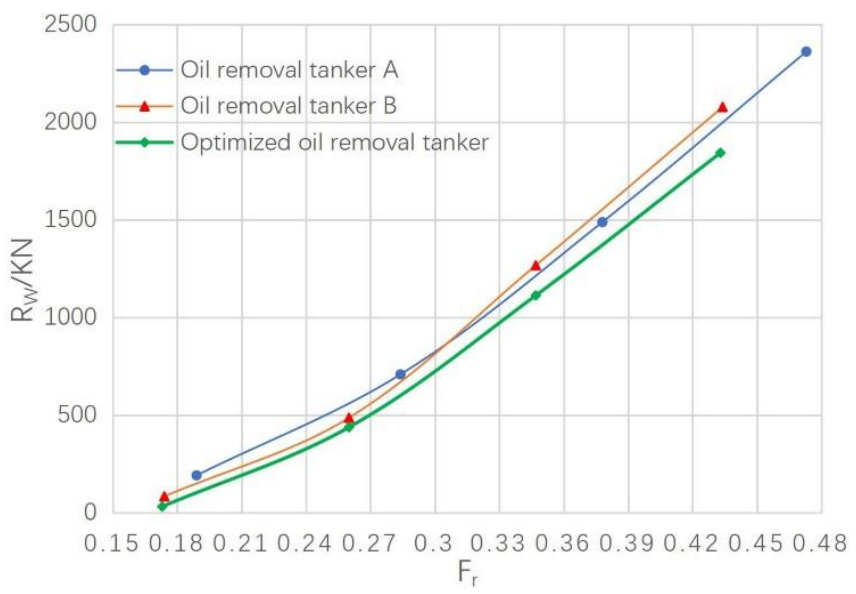

Figure 11: Wave resistance after optimization

When the tanker sails at medium and high speed and under the same working conditions, the wavemaking simulated by the plane of symmetry is compared with the tanker $\mathrm{A}$ and $\mathrm{B}$ before optimization, as shown in Figure 12: observing three-ship configuration of free surface wave, it can be seen that the flow field around the tanker interferes with the free surface, and the free surface is no longer a horizontal plane, but it arouses waves on the horizontal plane, generates vortices, and generates wave-making resistance. According to the observation of the pressure cloud diagram (Figure 13), the pressure value at the tail of the oil removing tanker is lower than that at the 
corresponding point in front of the submersible. The free surface at the tail of the oil removing tanker is sunken, and the free surface is stimulated to generate surface waves. At the same time, the high pressure of the fluid at the front end of the bow of the tanker makes the free surface at the bow of the tanker excite surface waves and generate wave resistance.

Combined with Figure 12, when sailing at medium and high speed, it can be seen that the wave-making near the bow of the optimized oil remover is relatively gentle, while the wave-making near the bow and tail of the oil remover A and B is obvious and large in amplitude, especially the bow will stimulate large surface waves. Compared with the tanker before optimization, the optimized tanker can greatly reduce the wave resistance, and the wave amplitude near the bow and tail is small.

It can be concluded since the optimized tanker itself has excellent overall shape and stronger drag reduction performance, it is verified that the optimization method based on the overall shape of the tanker proposed in this paper can reduce the wave-making resistance of the hull's free navigation surface[28][29].

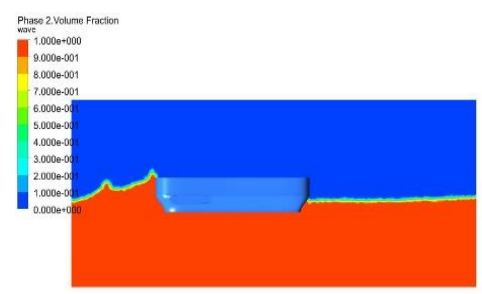

Oil removal vessel A

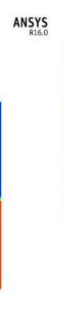

$\stackrel{亡}{\leftrightarrow}$

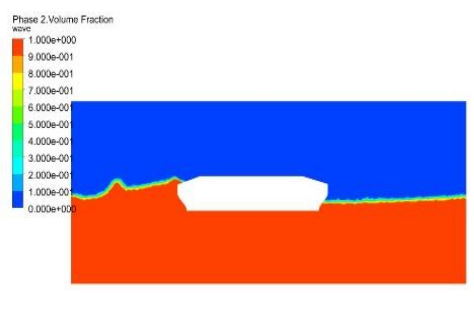

Oil removal vessel B

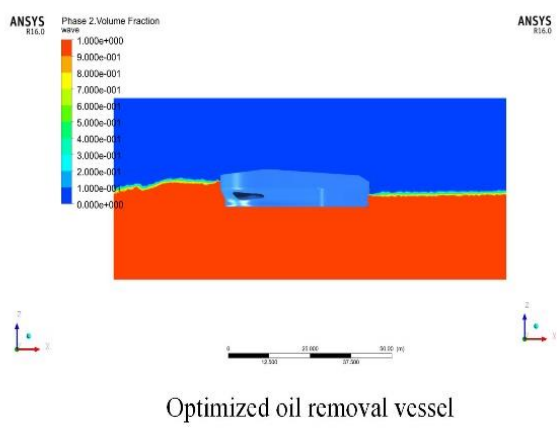

Optimized oil removal vessel

Figure 12: Comparison of wave-making on the symmetry plane of the tanker before and after tanker type optimization

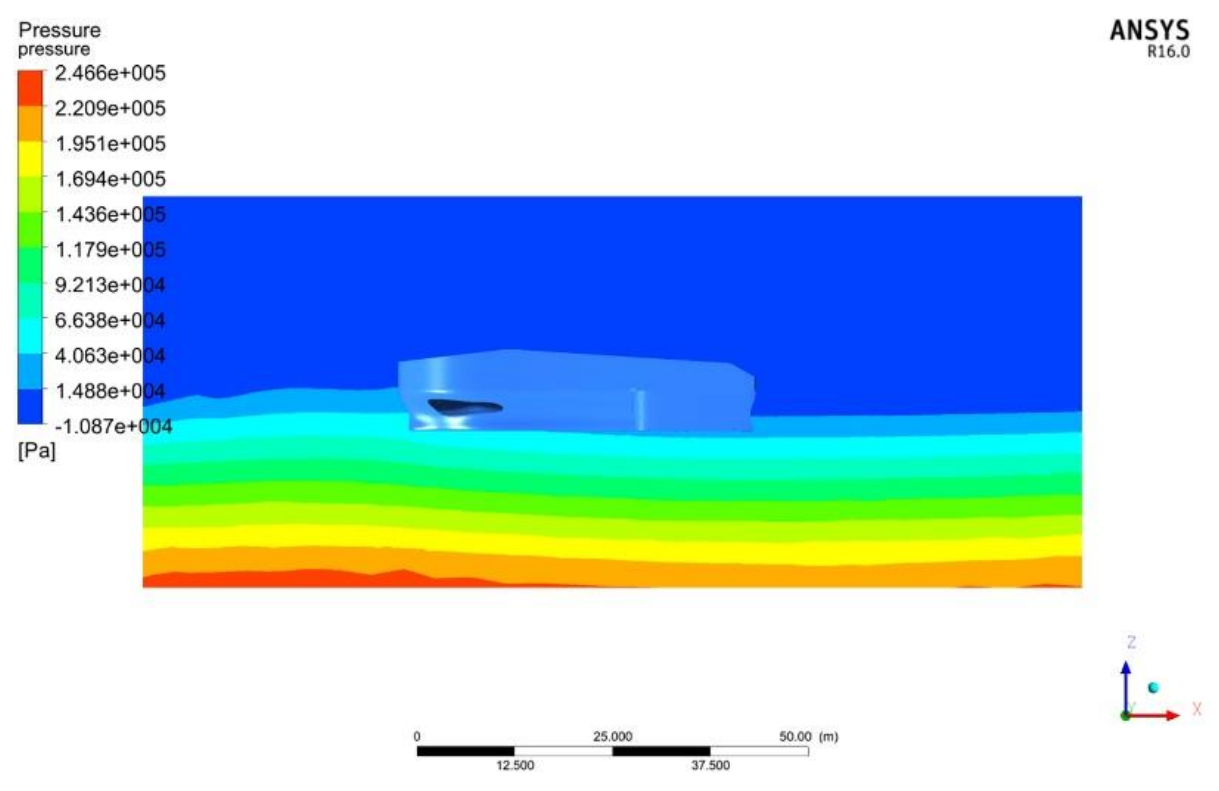

Figure 13: Pressure cloud map of the symmetrical plane of the optimized tanker

The correctness and accuracy of such calculations cannot be absolutely guaranteed. But it is similar to the analysis in other references [30][31], which shows that the calculation results of FLUENT software are very good. At the same time, this paper focuses on the comparison of the flow field of the three configurations of the oil removal tanker, so the calculation error generated by FLUENT can be ignored.

\subsection{Modelling rendering of the tanker after optimization}

The color matching design is carried out for the optimized modeling scheme of the oil-removing tanker, and the rendering design is carried out through the KEYSHOT software, and the threedimensional modeling effect as shown in Figure 14 is obtained. 


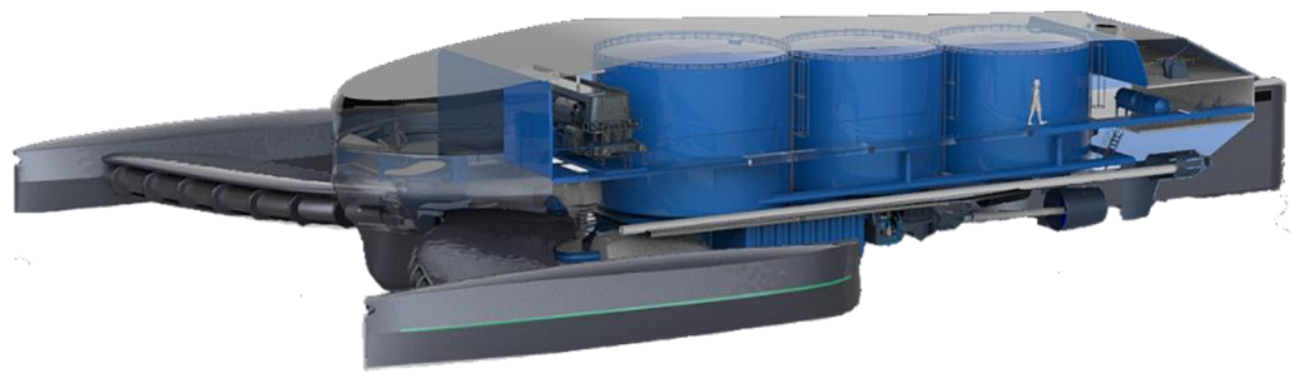

Figure 14: Modeling rendering scheme

\section{Conclusion}

(1) Aiming at the problems of low overall working efficiency and poor drag reduction performance of offshore oil remover during navigation, an optimization method based on overall shape of oil remover is proposed in this paper. In this paper, the computational fluid dynamics software FLUENT and VOF are adopted to build the multiphase flow numerical simulation model of the offshore oil removing tanker, and the flow field of the oil removing tanker during the sailing process is analyzed. It is calculated that the overall shape optimization of the oil removing tanker can reduce the wave resistance about $518.13 \mathrm{kN}$, and the maximum reduction is about $21.95 \%$, which greatly improve the efficiency of the tanker.

(2) The resistance coefficient $\mathrm{Cd}$ of the tanker before the optimization is relatively large, 0.307 0.606 in the given speed range. The overall optimization method of the oil removal tanker proposed in this paper can effectively improve the work efficiency and drag reduction performance, and greatly reduce the impact of wave-making resistance on the driving process of the tanker. After optimization, the resistance coefficient $\mathrm{Cd}$ of the tanker is reduced to $0.042-0.383$, which is a maximum reduction of 0.479 compared to before optimization.

(3) The computational fluid dynamics technology is used to study the hydrodynamic performance of ships, which greatly reduces the experimental cost. In this paper, the optimization method and the overall shape optimization scheme of the offshore oil removing tanker are proposed, which provides an important reference for the research and development of the offshore oil removing tanker.

\section{Acknowledgments}

The research is funded partially by the Jiangsu Province Policy Guidance Program-Subei Science and Technology Project (SZ-YC202001).

\section{References}

[1] Huang, L., Deng, S., Guan, J., Chen, M., and Hua, W.: Development of a novel high-efficiency dynamic hydrocyclone for oil-water separation, Chem. Eng. Res. Des., 130, 266-273, doi : 10.1016/j.cherd.2017.12.030, 2018.

[2] Chen, K. and Huang, D. B.: Application of CFD technology in research of resistance performance of trimaran, Journal of Harbin Engineering University, 27, 362-366, doi:10.3969/j.issn.10067043.2006.03.010, 2006.

[3] Zhu, L. C., Wang, D. G., and Gu, J. P.: Offshore oil spill and prevention, Shandong chemical industry, 47,88-89, doi: CNKI: SUN: SDHG. 0. 2018-23-032, 2018.

[4] Deng, R., Huang, D. B., and Li, J.: The FLUENT software is discussed from the numerical calculation of several types of ship resistance, Marine engineering, 32,55-59, doi: 10.3969/ j.issn.1000-6982.2010.02.015, 2010.

[5] Li, J. H. and Huang, D. B.: Study on the relationship between trimaran configuration and wave resistance, Journal of Harbin Engineering University, 26-32, doi: 10.3969/ j.issn.10067043.2002.01.006, 2002.

[6] Lu, C., Cui, J. Y., Meng, F. H., and Yu, K. T.: Multischeme Optimization Design of high speed Ship Type Fusion with special bulbous bow, Chinese Ship Research, 15,54-60, doi: 10.19693/ j.issn. 1673-3185.01556, 2020.

[7] Liu T. Q., Li Z. J., Shi G. M., Chen X. L., and Li Y. L.: A de-oiling vessel and method for cleaning slick oil on the surface using magnetic powder recovery, Liaoning Province, CN108951580B, 2020-04-24.

[8] Qin H. T.: Design and research of a trident-type oil-absorbing vessel, Henan Science and Technology, 126-127. doi: CNKI: SUN: HNKJ. 0.2018-05-053, 2018.

[9] Cheng, X. X., Ren, W., Song, X. F., and Liu, X. J.: Study on the structure design of dynamic cyclone separator for Ship oily wastewater, Marine engineering, 35,36-39, doi: CNKI: SUN: CANB.0.2013-05-012, 2013.

[10] Cheng X. X., Liu X., and Li Y.: A ship auxiliary oil purifier, Shandong Province, CN204952359U, 2016-01-13.

[11] Wang, X., Olsen, S. M., Martinez, E. A., Olsen, K. N., and Kiil, S.: Drag resistance of ship hulls: effects of surface roughness of newly applied fouling control coatings, coating water absorption, and welding seams, J. Coat. Technol. 
Res., 1-13, doi:10.1007/s11998-018-0054-7, 2018.

[12] Marinić-Kragić, I., Vučina, D., and Ćurković, M.: Efficient shape parameterization method for multidisciplinary global optimization and application to integrated ship hull shape optimization workflow, Ifac. Symp. Series., 80, 6175, doi:10.1016/j.cad.2016.08.001,2016.

[13] Xia, X. H. and Xu, S. J.: Numerical Simulation of ship-propeller Interference characteristics considering Free Surface, Journal of Zhejiang Ocean University (Natural Science edition), 33,185-189, doi:10.3969/j.issn.1008-830X. 2014. 02. 016, 2014.

[14] Wang, F. J.: Computational fluid dynamics analysis: Principle and application of CFD software, Tsinghua University Press, Beijing, 2004.

[15] Yu, L.: Study on the influence of trimaran configuration change on flow field and drag, M.S. thesis, College of Marine Engineering, Harbin Engineering University, 89pp., doi:10.7666/ d.y2054185, 2011.

[16] Zhang, J., Fang, J., and Fan, B. Q.: Review of VOF method theory and application, Progress in water conservancy and hydropower science and technology, 25, 67-70, doi: 10.3880/ j.issn. 10067647.2005.02.019, 2005.

[17] Lan, L.: Ship wave resistance calculation and profile optimization, M.S. thesis, College of Marine Engineering, Harbin Engineering University, 95pp., doi:10.7666/d.Y2236016, 2012.

[18] Jiao, J., Huang, S., and Soares, C. G.: Numerical investigation of ship motions in cross waves using CFD, Ocean. Eng., 223, 108711, doi: 10.1016/ j.oceaneng.2021.108711,2021.

[19] Duy, T. N., Hino, T., and Suzuki, K.: Numerical study on stern flow fields of ship hulls with different transom configurations, Ocean. Eng., 129, 401-414, doi:10.1016/ j.oceaneng. 2016. 10.052, 2017.

[20] Nazemian, A., Ghadimi, P.: CFD-based optimization of a displacement trimaran hull for improving its calm water and wavy condition resistance, Appl. Ocean. Res., 113, 102729, doi:10.1016/j.apor.2021.102729,2021.

[21] Zhao, T., Sun, P., and Yu, L.: Reliability of flow field numerical simulation of trimaran based on CFD, Journal of Huazhong University of Science and Technology (Natural Science Edition), 40,8183, doi:CNKI:SUN:HZLG.0.2012-11-016, 2012.
[22] Liu, Y. L.: Research on liner optimization of VLCS based on CFD, M.S. thesis, China Shipbuilding and Ocean Engineering Design and Research Institute, China Ship Research Institute, 111pp., doi:CNKI:CDMD:2.1014.069400, 2014.

[23] Lin, Y. and He, J. Y.: Prediction of ship resistance and free motion based on RANS method, Marine engineering, 41, 52-57+77, doi: CNKI: SUN: CANB.0.2019-12-012, 2019.

[24] Xiao M. H., Lin H., Guo S., Lin Y., Yang F., Zeng X. W., Zhang T. P., Geng G. S.: A de-oiler for offshore oil leakage, Jiangsu Province, CN211568243U, 2020-09-25.

[25] Xie, L. L., Chen, S. H., and Wu, J. P.: FLUENT software is used to optimize ship resistance performance, Journal of Huazhong University of Science and Technology (Natural Science Edition),37,101-103+124, doi:CNKI: SUN: HZLG. 0.2009-06-026,2009.

[26] Chen, S. L. and Yang, S. L.: Simulation of Viscous Flow around Surface Ship Using ANSYS-Fluent CFD Code, Appl. Mech. Mater., 419, 127-133, doi:10.4028/www.scientific.net/AMM.419.127,2 013.

[27] Wang, G. Y., Yu, D., Yin, M. L., Liu, G. J., Chen, J. T., and Li, Z. W.: Research on resistance performance of a research ship, Marine engineering, 39,45-48, doi: CNKI: SUN: CANB. 0. 2017-S1-014,2017.

[28] Zhang, Z. R., Liu, H., Zhu, S. P., and Zhao, F.: Application of CFD in ship engineering design practice and ship hydrodynamics, J. Hydrodyn., 18,315-322, doi:10.1016/S1001-6058(06) 60072 $-3,2006$

[29] Bakica, A., Gatin, I., Vukcevic, v., Jasak, h., and Vladimir, N.: Accurate assessment of shippropulsion characteristics using CFD, Ocean. Eng., 175, 149-162. doi:10.1016/ j.oceaneng. 2018.12.043,2019.

[30] Qi, D. Z., Chen, L., Zhou, M. G., Gong, Y., and Liu, M. Y.: Drag reduction performance analysis of ship tractor based on multiphase flow, Agricultural mechanization research, 41,264-268, doi:CNKI:SUN:NJYJ.0.2019-12-050,2019.

[31] Du, Y. W., Li, B., and Zhou, X. Y.: Design and optimization of wave-piercing catamaran based on resistance analysis, Ship science and Technology, 41,31-34+44, doi:10.3404/ j.issn. 1672-7649.2019.07.006,2019. 